\title{
Non-invasive screening of lung nodules in mice comparing a novel volumetric computed tomography with a clinical multislice CT
}

\author{
SUSANNE GRESCHUS ${ }^{1,5^{*}}$, RAJKUMAR SAVAI ${ }^{2 *}$, JOACHIM CLAUDIUS WOLF $^{3}$, \\ FRANK ROSE $^{2}$, WERNER SEEGER ${ }^{2}$, PAUL FITZGERALD $^{4}$ and HORST TRAUPE ${ }^{1}$ \\ Departments of ${ }^{1}$ Neuroradiology, ${ }^{2}$ Internal Medicine II, and ${ }^{3}$ Radiology, \\ Justus-Liebig-University, Giessen, Germany; ${ }^{4}$ GE Global Research Center, Schenectady, NY, USA
}

Received September 14, 2006; Accepted November 2, 2006

\begin{abstract}
In vivo imaging of small animal models will play an increasingly important role in cancer research, as new imaging systems that employ non-invasive protocols and offer high-resolution capability become available. A flatpanel volumetric computed tomograph (fpvCT) was evaluated to determine if minimally invasive protocols can be used to provide the spatial resolution required for lung imaging in small animals. The detection of small pulmonary nodules in a Lewis carcinoma model was investigated, and fpvCT was compared with a multislice computed tomograph (MSCT). Five C57/BL6 mice with Lewis lung carcinoma were monitored with both modalities over two weeks. Sensitivity of the systems was measured by comparing the results with histology, and the incidence of first visualization of the tumors in the two systems was determined. Compared to MSCT, fpvCT proved its superior sensitivity in detection of lung nodules. Due to its isotropic resolution and a significant reduction of partial volume effects, early detection and reasonable determination of growth in very small tumors was only possible with fpvCT. fpvCT is a high-resolution imaging system that proved its ability to perform in vivo monitoring of a pulmonary lung tumor model in mice. This permits longitudinal investigations in small animals for cancer research.
\end{abstract}

\section{Introduction}

Tumor models with human cell lines have now been developed, and novel therapeutic approaches such as antiangiogenic and

Correspondence to: Dr Susanne Greschus, ${ }^{5}$ Present address: Department of Radiology, University of Bonn, Sigmund-Freud-Str. 25, 53127 Bonn, Germany

E-mail: susanne.greschus@ukb.uni-bonn.de

${ }^{*}$ Contributed equally

Key words: carcinoma, Lewis lung, computed tomography, flat-panel detector, cancer monitoring genetic therapies are becoming available. These factors create an increased need for the development of high-resolution in vivo imaging technologies that can be applied to small animal models for tumor screening and volumetry. Longitudinal studies could play a significant role in cancer research, because these can provide information well beyond morpho$\operatorname{logy}$ at a single time-point. However, longitudinal studies should employ minimally invasive protocols. Therefore, imaging systems with high spatial resolution, short scan times, and low radiation dose are required.

High-resolution magnetic resonance imaging (MRI) systems have already been demonstrated for this purpose (1). Major disadvantages are long acquisition times and limited field-of-view, depending on the required resolution. Microcomputed tomography $(\mu \mathrm{CT})$ systems provide high spatial resolution, but the $\mathrm{x}$-ray technique required to produce image quality that allows utilization of this resolution introduces some risk of high radiation dose to the animal (2). Furthermore, the long acquisition times result in high susceptibility to motion artifacts, require a long duration of anesthesia, and prohibit measurement in apnea for the entire scan duration. Good image quality and high spatial resolution can be demonstrated for respiratory-gated and ex vivo investigations, but these strategies are complicated or impossible to use for longitudinal, intraindividual studies of tumor growth or therapy response $(1,3)$.

A recent review by Schuster et al (4) provides an overview of the available methods for small animal lung imaging. None of the tools are satisfactory for quantitative and longitudinal monitoring of small lung nodules. These disadvantages have now been overcome with the recently introduced flat-panel volumetric computed tomograph (fpvCT) (5). This provides $3 \mathrm{D}$ isotropic spatial resolution of up to $25 \mathrm{lp} / \mathrm{cm}$ at $10 \%$ MTF, which is adequate for studies of small structures such as pulmonary nodules in mice. Furthermore, the relatively short scanning time enables brief anesthesia with the option of follow-up investigations. The aim of our study was to optimize the scanning protocol in small animal lung investigations and to compare the fpvCT with a clinical multislice system. The sensitivity of both systems was evaluated by comparing the last scan of the animal with histological investigations, performed immediately following the scan. 


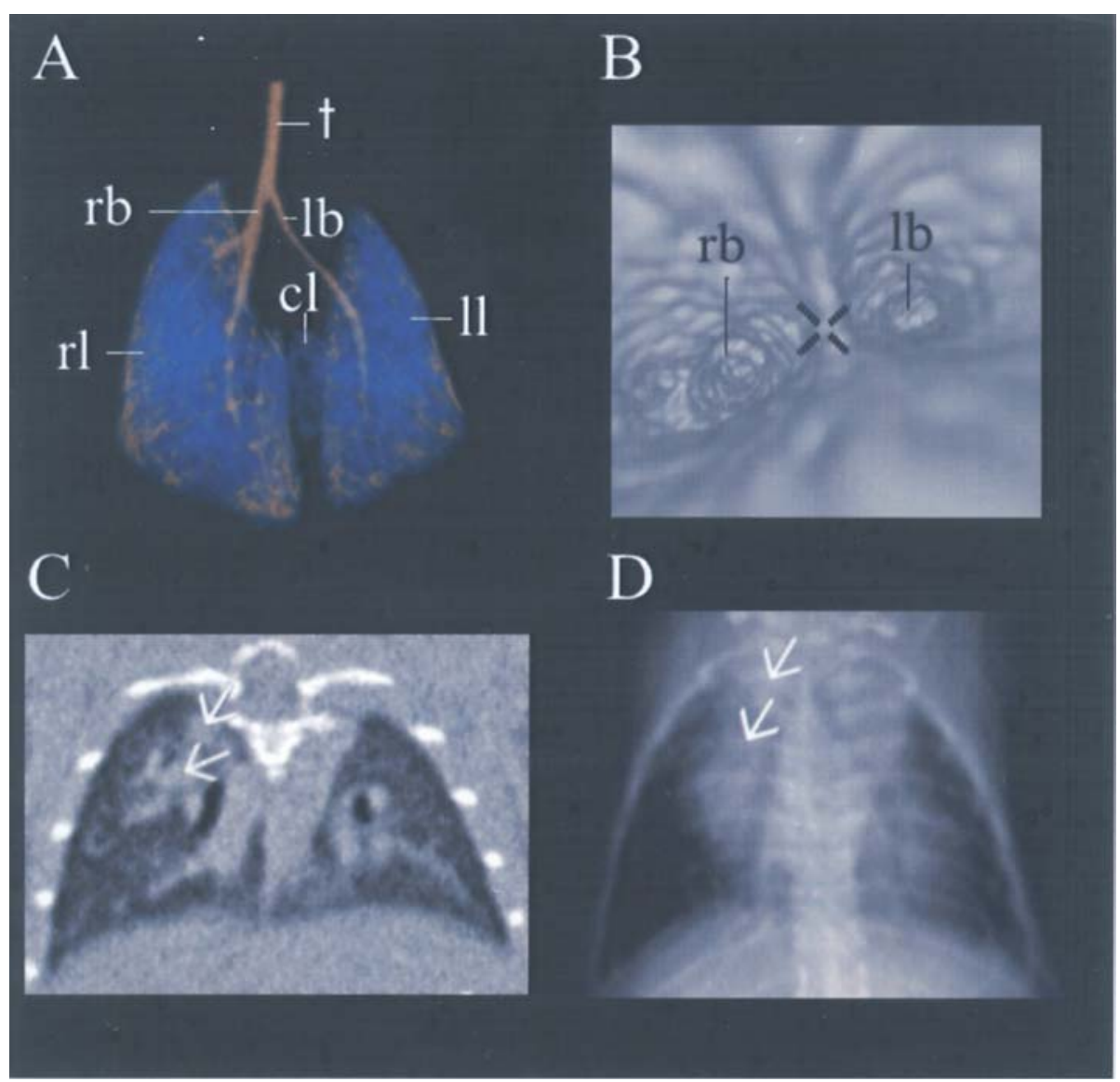

Figure 1. fpvCT images are shown in various display formats. In panel A, a 3D segmentation of the entire lung is shown. The trachea (t), left and right bronchus $(\mathrm{lb}, \mathrm{rb})$ and the division of the latter into its segmental bronchi are clearly delineated. The left lung (ll) consists of only one segment while the right lung (rl) has four segments, where the cardiac lobe (cl) extends over to the right side. With the 3D software, virtual bronchoscopy can be performed. In panel B, a view on the diversion of the trachea (cross) into the right bronchus (rb) and left bronchus (lb) is indicated. Single reformatted slices (coronal view, panel C) can be combined to a 3D dataset as demonstrated (panel D). The tumor masses (arrows in C and D) appear as an enlargement of the mediastinum, which correlates with human chest imaging.

\section{Materials and methods}

Animals. The experiments were performed in accordance with the National Institutes of Health Guidelines on the use of laboratory animals. Both the University Animal Care Committee and the Federal Authorities for Animal Research of the Regierungspräsidium Giessen (Hessen, Germany) approved the study protocol. Five adult (5-7 weeks) C57BL/6N mice (Charles Rivers, Germany) were inoculated with Lewis lung carcinoma (LLC1) via intratracheal injection of $1 \times 10^{6}$ cells. LLC1 cells were obtained from American Type Culture Collection (ATCC, Manassas, VA). The cells were routinely cultured in tissue culture flasks containing RPMI-1640 medium (PAN biotech $\mathrm{GmbH}$, Germany) supplemented with 2\% FBS (Fetal Bovine Serum, Greiner bio one, Germany), penicillin (100 U/ml), and streptomycin (0.1 mg/ml; Gibco, Germany), maintained at $37^{\circ} \mathrm{C}$ in a humidified atmosphere containing $5 \% \mathrm{CO}_{2}$ in air. For scanning, the animals were anesthetized with an intraperitoneal injection of 0.05-0.1 ml solution containing $0.1 \mathrm{ml}$ Rompun $2 \%$ (Bayer, Leverkusen, Germany), $0.1 \mathrm{ml} \mathrm{Ketavet} 100 \mathrm{mg} / \mathrm{ml}$ (Pharmacia GmbH, Erlangen, Germany) and $0.2 \mathrm{ml} \mathrm{NaCl}$. The mice were investigated with both scanners using the same anesthesia (6). All cell culture studies and animal experiments were performed by the author (RS).
Multislice computed tomography. MSCT imaging was performed on a Somatom Plus 4 Volume Zoom scanner (Siemens, Erlangen, Germany) using $120 \mathrm{kV}$ and $320 \mathrm{mAs}$, a technique equivalent to that used for the fpvCT. For image reconstruction a high-resolution kernel was applied (U90). Images with $0.5-\mathrm{mm}$ slice thickness and $0.5-\mathrm{mm}$ spacing were acquired in a step and shoot scanning mode (7).

Volumetric computed tomograph. The volumetric computed tomograph (fpvCT) was developed by General Electric Global Research Center (Niskayuna, NY, USA). In this experimental system, a clinical CT X-ray tube is mounted on a standard rotating gantry. The X-ray source, which can be operated over the range of $70-140 \mathrm{kVp}$, is collimated to produce a cone beam, which irradiates an amorphous silicon flat-panel detector with a $1024 \times 1024$ matrix of $200 \mu \mathrm{m}$ pixels. The subject is placed on a sample holder that is mounted to a standard patient table, and is stationary during the acquisition, while the gantry rotates around it. Rotation times can range from 2 to 8 sec, and 500-2000 views can be acquired during one rotation. One gantry rotation typically covers $4.3 \mathrm{~cm}$ in the $\mathrm{z}$ direction. For additional $\mathrm{z}$ coverage, the table translates further into the gantry bore for each rotation $(5,7)$. Images are reconstructed using a cone-beam algorithm with various kernel options, into an arbitrary matrix size of arbitrary voxel sizes. System 


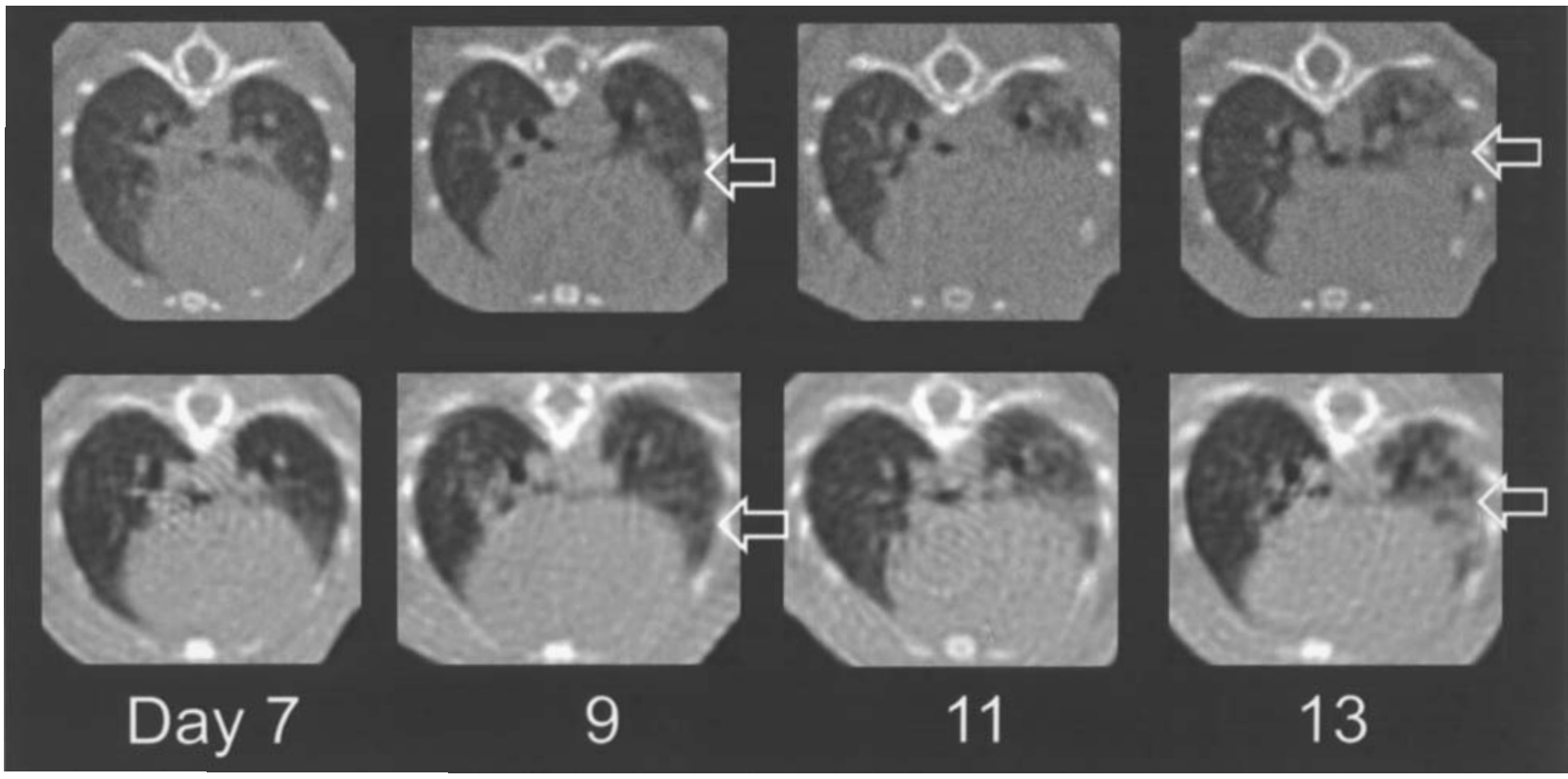

Figure 2. Axial slices acquired with fpvCT (upper row) and MSCT (lower row). The fpvCT shows a better image quality with less noise in the images. Though the animal was freely breathing, the spine and ribs were much better delineated in fpvCT than in MSCT. The small nodule detected in fpvCT on day 9 is not visible in the corresponding image from MSCT (arrows). For the nodules seen with both modalities, a superior delineation of the tumor border is presented in fpvCT.

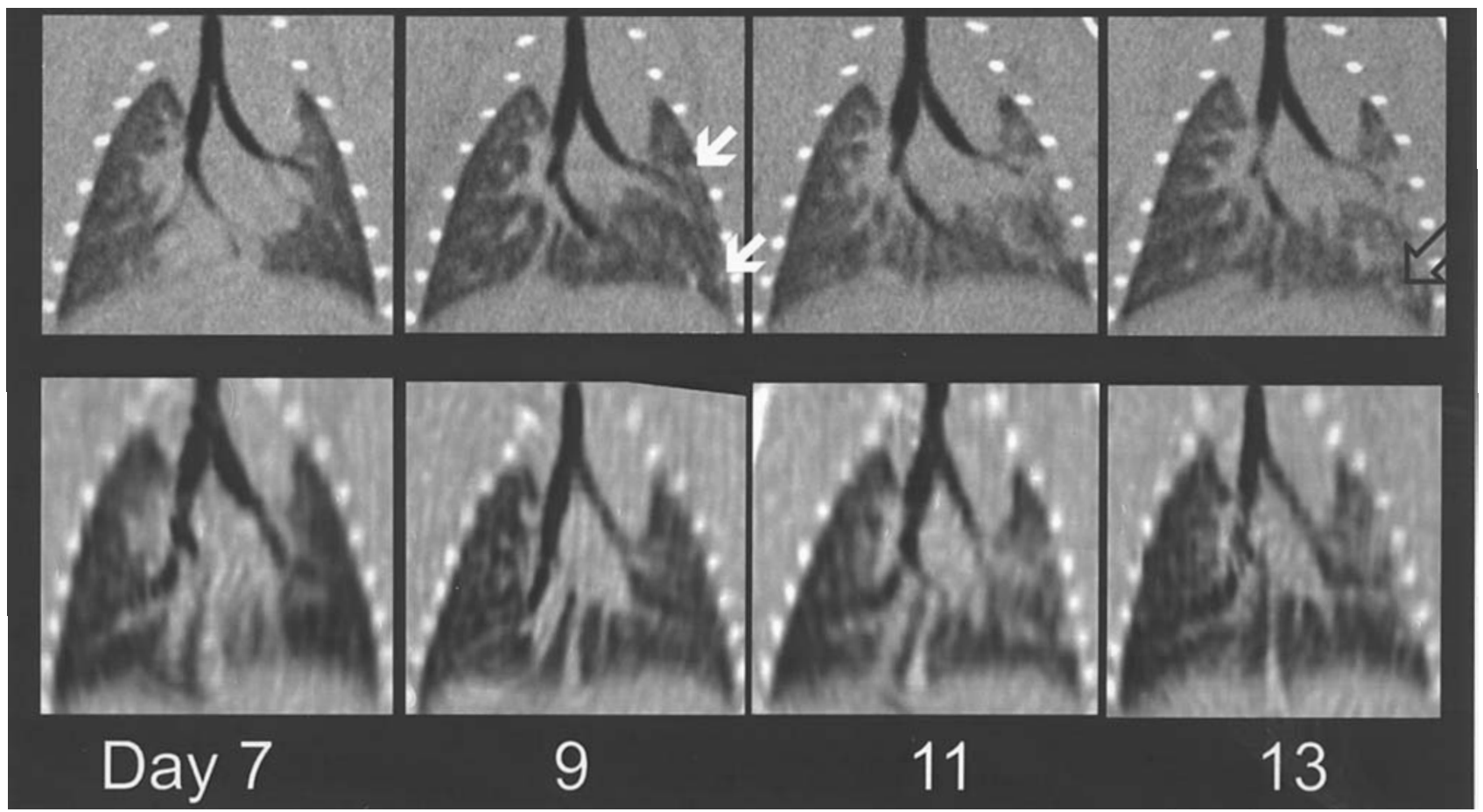

Figure 3. Display of coronal reformatted images of datasets acquired with fpvCT (upper row) and MSCT (lower row). The first tumor nodules were detected with fpvCT on day 9 (white arrows), while these nodules were not visible at that time in MSCT images. Tumors were visible at day 11 in MSCT images, although the small nodules were still not visible in later stages, as they were in fpvCT images (open arrow). All tumors were much better delineated in fpvCT images and at all time-points. The coronal reformatted images from MSCT appear to be blurred, which is caused by anisotropy (less resolution in the z direction than in plane).

spatial resolution has been measured to be $25 \mathrm{lp} / \mathrm{cm}$ at $10 \%$ MTF using a $25-\mu \mathrm{m}$ tungsten wire.

For this study, $120 \mathrm{kVp}$ and $40 \mathrm{~mA}$ were used, and 1000 views were acquired in an 8 -sec rotation. A single rotation covered $4.3 \mathrm{~cm}$, sufficient for the mouse thorax. All reconstructions used the edge-defining kernel and $50 \mu \mathrm{m}^{3}$ voxels. All data were transferred to an Advantage Workstation 4.1 (General Electric HealthCare Europe, Buc, France) and processed with volume rendering software (Voxtool, version $3.058 \mathrm{c})$. 
Correlation between $f p v C T$ and MSCT. The animals were scanned before tumor induction and on days 3,5,7,9,11,13. Two observers (SG,JW) assessed the scans in consensus, blinded to the image source (modality and time point). Axial slices of each follow-up scan were compared with the baseline (before tumor induction). Tumor diameter and location were recorded.

Correlation between fpvCT, MSCT and histology. To evaluate the sensitivity of the imaging systems using histology as a gold standard, the lungs were harvested and investigated in histology after hematoxylin and eosin staining immediately following the last scan. For better orientation and correlation coronal slices were used. One investigator (RS) evaluated the results, blinded to the scanning results.

\section{Results}

Images from both modalities were free of major motion artifacts; overall image quality was therefore found to be adequate for both. Comparing axial slices from the two systems, higher spatial and therefore anatomical resolution was obvious in fpvCT images. Bronchial structures were clearly delineated and distinguished from pulmonary arteries, pulmonary veins and lung parenchyma in fpvCT images. The bronchial system and the whole lung could be tracked from its outlet for three-dimensional segmentation (Fig. 1). The four segments of the right lung could vaguely be distinguished in fpvCT, especially in cases of pleural effusion, while there was no differentiation possible with MSCT. Pulmonary nodules were far better delineated in fpvCT compared to the clinical scanner. Small nodules, which were frequently attached to pulmonary arteries, were most easily detected in coronal reformatted images. Since fpvCT produces isotropic resolution, equally high resolution was permitted for axial images or any reformatted slice (Figs. 2 and 3). Therefore a better delineation and detection of tumors in coronal planes was possible, as the nodules could be distinguished from the pulmonary arteries, which were visualized from their origin in the pulmonary artery to the periphery. The tumors showed preferable growth along the pulmonary arteries and were therefore more readily observed in coronal slices.

In Table I the time-point of lung nodule detection is stated in MSCT versus fpvCT and finally the results on the last scan are opposed to investigation in histology. Tumor inoculation was successful in four mice (Mouse 2 to Mouse 5 in Table I), visualized in longitudinal imaging and proven in histology. Both imaging modalities revealed a good overall specificity of $100 \%$. In one mouse (Mouse 4 ) on day 9 tumor growth was detected in fpvCT, but this could not be proven in the following scan. Therefore probably infiltrate or atelectasis was mistaken for tumor tissue. Comparing the scans on day 11 with the subsequently performed histology, scanning with MSCT resulted in a sensitivity of $50 \%$ compared to $83 \%$ in fpvCT (calculated separately for the left and right lung. Therefore, MSCT was $60 \%$ as sensitive as fpvCT $(.50 / .83=$ .6). Because no mice were sacrificed prior to the end of the study, images taken prior to day 11 could not be compared with ex vivo investigation, and therefore a gold standard was not available for these time-points. The only possible comparison
Table I. Lung nodule detection in MSCT versus fpvCT.

\begin{tabular}{lccccccc}
\hline Mouse & & Day 3 & Day 5 & Day 7 & Day 9 & Day 11 & Histology \\
\hline 1 & $\begin{array}{c}\text { left } \\
\text { right }\end{array}$ & & & & & & \\
& & & & & & & \\
2 & left & & & + & + & + & $\bullet$ \\
& right & + & + & + & + & $\oplus$ & $\bullet$ \\
3 & left & & & & & & \\
& right & & & + & $\oplus$ & $\oplus$ & $\bullet$ \\
4 & left & & + & $\oplus$ & $\oplus$ & $\oplus$ & $\bullet$ \\
& right & & & & + & & \\
5 & left & & & & + & + & $\bullet$ \\
& right & & & & & & $\bullet$ \\
\hline
\end{tabular}

The results for the left and right lung of the five mice are tabulated for each day of investigation with fpvCT and MSCT, and finally for histology on the 11th day after tumor implantation. + indicates nodules were detected with fpCT but not MSCT, $\oplus$ indicates nodule detection with both fpvCT and MSCT, and the presence of nodules found with histology is shown by $\bullet$. Except for Mouse 4, all tumors were detected earlier in fpvCT. Sensitivity was superior in fpvCT compared to MSCT in the end-point of the investigation, while the specificity was $100 \%$ for both systems.

was between MSCT and fpvCT. All tumors detected in both modalities could be followed in their growth and were confirmed in the final investigation with histology. However, confirmation of tumor inoculation was provided earlier by fpvCT. We found that in relative terms, sensitivity in MSCT compared to fpvCT was $50 \%$ when calculated for each lung and day of scanning.

In addition to tumor monitoring and proof of successful tumor inoculation, side effects from tumor growth like pleural effusions and complications of intubation and tumor instillation were detectable. Pulmonary infiltrates in the early course of the disease and pneumo-mediastinum and pneumothorax were observed.

Correlating the histological slices with images from fpvCT, we estimate the lower limit on tumor detectability to be $0.2 \mathrm{~mm}$ in diameter (Fig. 4). However, it is possible that the shrinking that results from histological preparation might have influenced this result. Volumetric measurements can be easily obtained from the 3D fpvCT datasets, if there is good delineation between the tumors and any blood vessels to which they may be attached (Fig. 5). In our intratracheally induced tumor model a distinct delineation of tumor against pulmonary vessels is only vaguely possible, or sometimes impossible. An easier approach is the volumetric measurement of peripheral tumors that are well delineated from the surrounding tissue as seen in metastatic tumor models.

\section{Discussion}

Only a few reports about longitudinal studies of lung disease in mice can be found in medical literature $(1,8)$. Although there is an increasing need for methods to investigate tumor 


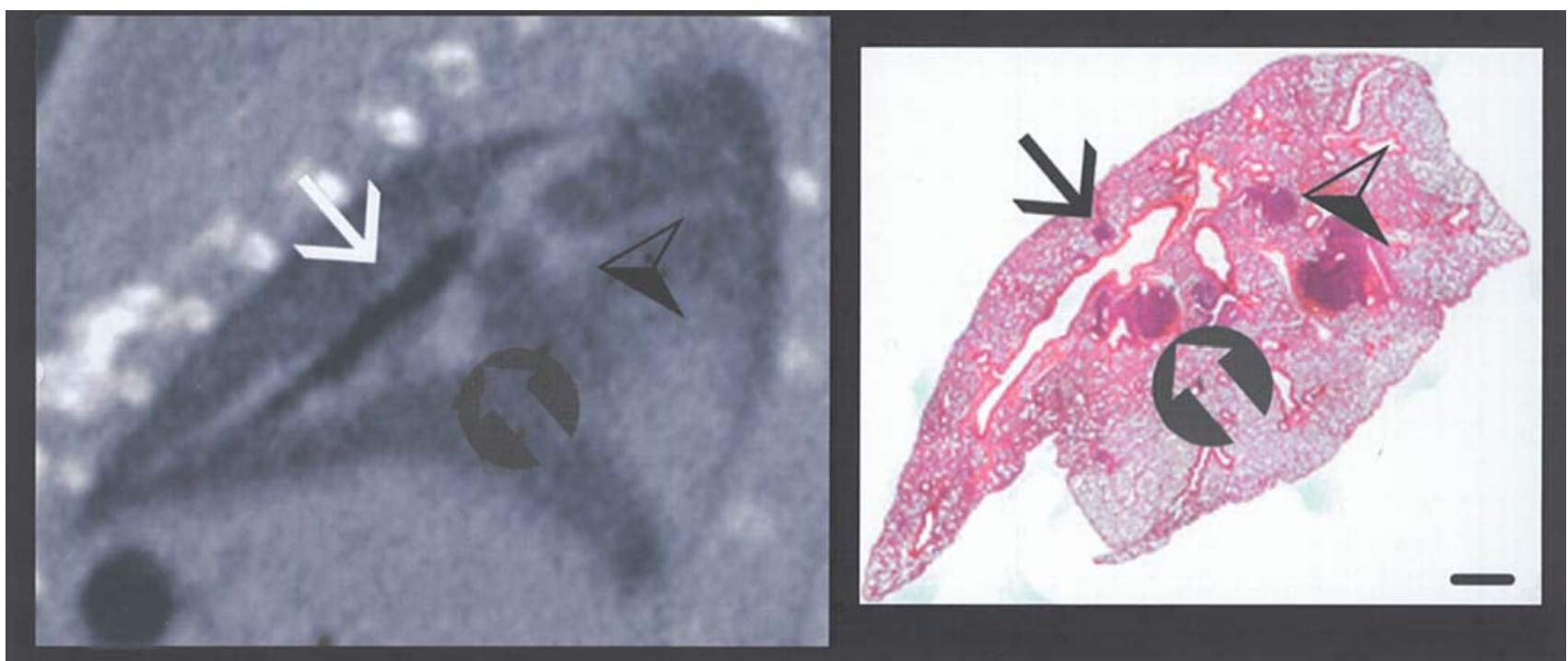

Figure 4. A comparison between an fpvCT image (left) and histology (right) is displayed. The small tumor adjacent to the bronchus (solid arrow) was measured to be $200 \mu \mathrm{m}$ in diameter in histology, but is only vaguely seen in fpvCT. Therefore, this seems to be the lower limit for detection of lung nodules in fpvCT. Larger nodules could easily be identified in fpvCT (open arrow, arrow head).

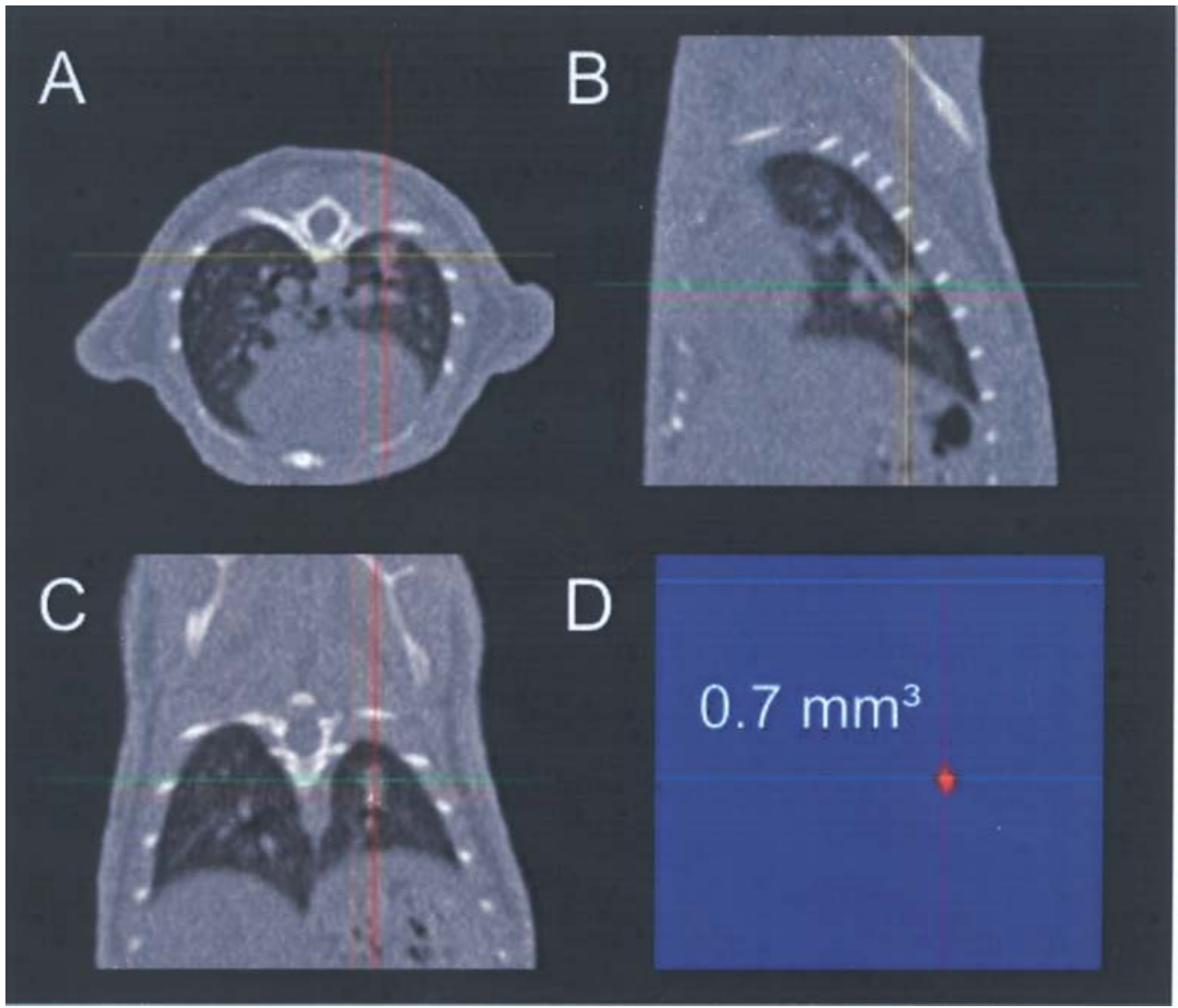

Figure 5. In this panel a three-dimensional reconstruction of a lung nodule from fpvCT data is shown in the axial (A), sagittal (B) and coronal (C) planes. There is no obvious difference in resolution in the three planes. This nodule was measured to be $0.7 \mathrm{~mm}^{3}$. A semi-automated threshold-based algorithm was used for segmentation in the post-processing software.

models, the lung is difficult to assess with conventional imaging techniques $(4,9)$, where a limiting factor is susceptibility to motion artifacts. The most promising modality is computed tomography, offering short scanning times and satisfactory soft tissue resolution in the lung due to the high contrast.
Short scanning times permit investigations in a short breath hold, using intubation. However, intubation is a very invasive procedure, which introduces risk of harming the animals and compromising the study, especially when investigating a pulmonary disease model with repeated scanning. To avoid 
this situation, we investigated the animals freely breathing. Thoracic movement was minimized by use of anesthesia with a muscle relaxant. No motion artifacts that interfered with image evaluation were observed in either fpvCT or MSCT, though slight blurring of the diaphragm indicated residual movement.

Compared to MSCT, fpvCT demonstrated a significant increase in sensitivity to small lung nodules. Therefore, fpvCT is useful for cancer research in small animals, to provide important information on lung cancer models. This information is (i) early verification of successful tumor induction, (ii) quantification of tumor growth, (iii) observation of possible complications (pneumonia, atelectasis) and most importantly (iv) evaluation of response to therapeutic approaches.

In addition to lung nodule monitoring, fpvCT is suitable for detection and characterization of structural lung pathologies such as emphysema or fibrosis (7). Furthermore, fpvCT has the ability to obtain functional information such as tumor perfusion, which was already reported for other modalities in subcutaneous tumors $(10,11)$. Morphological imaging of angiogenesis in a subcutaneous tumor model has been demonstrated (5) to be feasible with fpvCT, but visualization of the vessels within lung tumors is much more challenging, due to thoracic motion and the small tumor size. However, it would be helpful to get an indirect indication of angiogenesis in an orthotopic tumor model using perfusion imaging, especially to study anti-angiogenic approaches in tumor therapy (12).

In combination with other functional imaging modalities, fpvCT would be valuable to locate and quantify tumors. FpvCT could provide high-resolution morphological information that complements the functional information obtained from tomographic modalities with less resolution, such as PET and SPECT (13-17), or planar modalities such as optical imaging (18-21).

In conclusion, this study demonstrates that the flat-panel volumetric computed tomograph is a novel tool that proved its feasibility in detection and quantification of lung nodules in mice. Therefore it is a promising, valuable tool in cancer research. In our study, superiority to MSCT was clearly demonstrated. For future development, functional investigations with fpvCT would be helpful to evaluate angiogenesis in pulmonary nodules and potential success of antiangiogenic therapeutics.

\section{References}

1. Kennel SJ, Davis IA, Branning J, Pan H, Kabalka GW and Paulus MJ: High resolution computed tomography and MRI for monitoring lung tumor growth in mice undergoing radioimmunotherapy: correlation with histology. Med Phys 27: 1101-1107, 2000
2. Ford NL, Thornton MM and Holdsworth DW: Fundamental image quality limits for microcomputed tomography in small animals. Med Phys 30: 2869-2877, 2003.

3. Cavanaugh D, Johnson E, Price RE, Kurie J, Travis EL and Cody DD: In vivo respiratory-gated micro-CT imaging in smallanimal oncology models. Mol Imaging 3: 55-62, 2004.

4. Schuster DP, Kovacs A, Garbow J and Piwnica-Worms D: Recent advances in imaging the lungs of intact small animals. Am J Respir Cell Mol Biol 30: 129-138, 2004.

5. Kiessling F, Greschus S, Lichy MP, et al: Volumetric Computed Tomography (VCT): a new technology for noninvasive, highresolution monitoring of tumor angiogenesis. Nat Med 10: 1133-1138, 2004.

6. Savai R, Wolf JC, Greschus S, et al: Analysis of tumor vessel supply in Lewis lung carcinoma in mice by fluorescent microsphere distribution and imaging with micro- and flat-panel computed tomography. Am J Pathol 167: 937-946, 2005.

7. Greschus S, Kiessling F, Lichy MP, et al: Potential applications of flat-panel volumetric CT in morphologic and functional small animal imaging. Neoplasia 7: 730-740, 2005.

8. Plathow C, Li M, Gong P, et al: Computed tomography monitoring of radiation-induced lung fibrosis in mice. Invest Radiol 39: 600-609, 2004.

9. Mitzner W, Brown R and Lee W: In vivo measurement of lung volumes in mice. Physiol Genomics 4: 215-221, 2001.

10. Krix M, Kiessling F, Vosseler S, et al: Sensitive noninvasive monitoring of tumor perfusion during antiangiogenic therapy by intermittent bolus-contrast power Doppler sonography. Cancer Res 63: 8264-8270, 2003

11. Kiessling F, Krix M, Heilmann M, et al: Comparing dynamic parameters of tumor vascularization in nude mice revealed by magnetic resonance imaging and contrast-enhanced intermittent power Doppler sonography. Invest Radiol 38: 516-524, 2003.

12. Kerbel RS: Antiangiogenic drugs and current strategies for the treatment of lung cancer. Semin Oncol 31: 54-60, 2004.

13. Weissleder R, Cheng HC, Marecos E, Kwong K and Bogdanov A Jr: Non-invasive in vivo mapping of tumour vascular and interstitial volume fractions. Eur J Cancer 34: $1448-1454,1998$.

14. Lewis JS, Achilefu S, Garbow JR, Laforest R and Welch MJ: Small animal imaging: current technology and perspectives for oncological imaging. Eur J Cancer 38: 2173-2188, 2002.

15. Kiessling F, Farhan N, Lichy MP, et al: Dynamic contrastenhanced magnetic resonance imaging rapidly indicates vessel regression in human squamous cell carcinomas grown in nude mice caused by VEGF receptor 2 blockade with DC101. Neoplasia 6: 213-223, 2004

16. Budinger TF, Benaron DA and Koretsky AP: Imaging transgenic animals. Annu Rev Biomed Eng 1: 611-648, 1999.

17. Bremer C, Tung $\mathrm{CH}$ and Weissleder R: Molecular imaging of MMP expression and therapeutic MMP inhibition. Acad Radiol 9: S314-S315, 2002.

18. Yang M, Baranov E, Li XM, et al: Whole-body and intravital optical imaging of angiogenesis in orthotopically implanted tumors. Proc Natl Acad Sci USA 98: 2616-2621, 2001.

19. Ntziachristos V, Bremer C and Weissleder R: Fluorescence imaging with near-infrared light: new technological advances that enable in vivo molecular imaging. Eur Radiol 13: 195-208, 2003.

20. Mahmood U and Weissleder R: Near-infrared optical imaging of proteases in cancer. Mol Cancer Ther 2: 489-496, 2003.

21. Bremer C, Ntziachristos V and Weissleder R: Optical-based molecular imaging: contrast agents and potential medical applications. Eur Radiol 13: 231-243, 2003. 